\title{
Evaluation of tumour infiltrating lymphocytes in core needle biopsies and resected specimens of breast carcinoma
}

\author{
Parinitha S Sangam ${ }^{1}$, Priyanka Parmesh ${ }^{1}$ \\ ${ }^{1}$ Department of Pathology, Shri Dharmasthala Manjunatheshwara College of Medical Science and Hospital, Karnataka, India
}

\author{
Keywords: \\ Breast carcinoma; \\ Core needle biopsies; \\ HER2neu; \\ Tumor-infiltrating \\ lymphocytes;
}

\begin{abstract}
Background: Tumour-infiltrating lymphocytes are important predictors of response to neoadjuvant therapy in breast cancer. It remains unclear whether Tumour infiltrating lymphocytes scores in core needle biopsies are closely representative of those in the whole tumour of surgically resected specimens.

The study aims to evaluate the concordance between tumor-infiltrating lymphocytes scores of core needle biopsies and surgically resected specimens as per recommendations and to assess the reliability of tumorinfiltrating lymphocytes score in core needle biopsies.

Materials and Methods: Retrospective study from January 2016 to March 2019 done in tertiary care hospital. Tumor-infiltrating lymphocytes scores were sub-classified as low $(\leq 10 \%)$, intermediate (11$49 \%$ ), and high (40-90\%). Relevant statistical tests were used and reliability score was done by Fisher's test.

Results: The mean value of tumor-infiltrating lymphocytes in 34 cases was $27.03 \%$ in core needle biopsies and $34.6 \%$ in surgically resected specimens. High tumor-infiltrating lymphocytes score was seen in $8(23.6 \%)$ and $11(32.4 \%)$ cases and low to intermediate score was seen in $26(76.4 \%)$ cases and $23(67.6 \%)$ in core needle biopsies and surgically resected specimens respectively. Intermediate and high tumor-infiltrating lymphocytes categories in core needle biopsies accounted for good agreement with surgically resected specimens (60\%-intermediate, $87.5 \%$-high). More than three cores had a better agreement.
\end{abstract}

Conclusions: Tumor-infiltrating lymphocytes score in core needle biopsies is of reliable value in breast cancer. Discrepancies may occur in tumours with HER2neu phenotype, PR status, and younger age.

\section{Correspondence:}

Dr Priyanka Parmesh

Department of Pathology, Shri Dharmasthala Manjunatheshwara College of Medical Science and Hospital, Karnataka

ORCID ID: 0000-0002-9386-9308

Email:drpriyankamahesh@gmail.com

Reveived : July $23^{\text {rd }} 2020$; Accepted : September $2^{\text {nd }} 2020$

Citation: Sangam PS, Parmesh P. Evaluation of tumour infiltrating lymphocytes in core needle biopsies and resected specimens of breast carcinoma. J Pathol Nep 2020;10: 1711-7. DOI: 10.3126 jpn.v10i2.30227

Copyright: This is an open-access article distributed under the terms of the Creative Commons Attribution 4.0 International License, which permits unrestricted use, distribution, and reproduction in any medium, provided the original author and source are credited.

\section{INTRODUCTION}

The immune system has a crucial role to play in the disease outcome of patients with cancer. ${ }^{1}$ Several factors like age, histological type, grade, tumour size, lymph node status, hormone receptor, and HER2neu status and vascular invasion provide a variable prognosis on the survival of breast carcinoma patients. ${ }^{2}$ Research studies have proven that a relationship lies between tumour and host inflammatory response and its influence on the prognosis and metastasis of cancer. ${ }^{3,4}$ Tumour infiltrating lymphocytes (TILs) has been recognized as a biomarker of an antitumor immune 
response..$^{5-8}$ Recently it is found that TILs and microvessel density have a role in modifying the prognosis and survival of breast cancer patients. ${ }^{9} 10$ However, the literature survey suggests inconsistency about the results in the literature due to the absence of methodical validation concerning the outcome of TILs in breast carcinoma. ${ }^{11}$ In order to streamline this, international guidelines for evaluation of TILs in breast cancer was prepared by the International working group for TILs 2014. The aim was to standardize the evaluation of TILs based on Haematoxylins and eosin (H\&E) stained sections of core needle biopsies (CNB) and surgical resection specimen (SRS). After the guidelines were laid, many studies were conducted and observed that increase TILs are predictive of a better response to neoadjuvant therapy (NAT) and overall survival. ${ }^{12-19}$

In an era of NAT, where NAT has shown to have better clinical response and survival, the importance of CNB has routinely come into practice as it determines the specific NAT required. ${ }^{20}$ Studies have shown that CD8+ T lymphocytes and FOXp3+ cell comprise the breast cancer TILs. CD8+ cell activation causes autoregression of larger tumours. FOXp3+ cells are known to inactivate the CD $8+$ $\mathrm{T}$ cells. Studies show that the immune modulator used in NAT determines the survival of breast cancer patients. ${ }^{21}$ Thus, assessment of TILs and type of TILs helps in the introduction of an immune modulator in NAT. When CNB and SRS were studied concerning estrogen receptor (ER), progesterone receptor (PR), HER2neu receptor, and Ki67 disagreement were confounded. ${ }^{22,23}$ There are limited studies for TILs correlation between CNB and SRS. The present study was designed to compare the TILs status between CNB and SRS of breast carcinoma and to assess the reliability of the TILs score in CNB.

\section{MATERIALS AND METHODS}

It is a retrospective study from January 2016 to March 2019 conducted in the department of pathology at a tertiary care centre in southern India. 34 cases of breast carcinoma cases for which both CNB and SRS were obtained were included. Patients who received neoadjuvant chemotherapy, metastatic breast carcinoma, and recurrent carcinoma were excluded from the study group. Clinical, pathological, and treatment details with follow-up were obtained from department archives and computerized medical records.

Routine tissue processing on formalin-fixed tissues of CNB and SRS was performed and stained with H\&E stain. H\&E slides were double-blinded and TIL score was done by two pathologists according to the international guidelines for the assessment of TIL 2014..$^{24}$ Areas outside the tumour border, around the intraductal component and normal lobules, TILs with crush artifacts and necrosis were not considered. TILs included only lymphocytes and plasma cells. The average score was represented as a percentage. TIL scores were sub-classified as low $(\leq 10 \%)$, intermediate $(11-39 \%)$, and high (40-90\%). ${ }^{24}$ For simplifying the results and for better understanding, the TILs score were also assessed by twotier classification low/intermediate $(<40 \%)$ and high $(40 \%$ $90 \%$ ). In the $\mathrm{CNB}$, all cores containing invasive tumour cells were examined. Immunohistochemistry was performed on 4 micron thick sections. The primary antibodies used were ER, PR, HER2neu, and Ki67. Allred score was used to quantify the hormonal status. HER2neu staining was assessed as per the American Society of Clinical Oncology/ College of American Pathologists guidelines. Ki67 was assessed as an average number of positive cells for 1000 tumour cells and then reported as a percentage.

\section{Statistical methods}

Data were analyzed using SPS (v.20.0; SPSS, IBM Corporation, Bangalore, India). P-values of $<0.05$ were considered to be significant. The TIL scores of CNB and SRS were compared using paired t-tests and Pearson's correlation. Pearson's r-correlation was used to compare scores between CNB and SRS. Other relevant statistical tests like Fisher's test and ANOVA (one way) were applied to compare the results. The distribution and agreement of TIL score between CNB and SRS were done using the kappa statistical test. Values of $\kappa>0.6$ were correlated with good agreement, values between 0.4 and 0.6 considered moderate agreement, values $<0.4$ corresponded to fair, and values $<0.2$ reflected poor agreement. Univariate analysis was applied for risk factors affecting the TIL score based on 2 tier classification.

\section{RESULTS}

Out of 87 cases that underwent modified radical mastectomy during the study period, CNB was obtained for 34 cases. The mean age of the patient was 55 years (range 28 to 73 years). Modified radical mastectomy was done in all cases. Tumour size was pT1 $(<2 \mathrm{~cm})$ in 3 cases $(8.8 \%)$, pT2 $(2-5 \mathrm{~cm})$ in 13 cases $(38.3 \%)$, more than $5 \mathrm{~cm}$ in 15 cases $(44.1 \%)$ and Tx were 3 cases $(8.8 \%)$. The majority of the cases were infiltrating ductal carcinoma $(n=31,91.1 \%)$ and the remaining 3 cases $(8.8 \%)$ ) were infiltrating lobular carcinoma, medullary carcinoma, and tubular carcinoma each. Most of the cases, under Elston Ellis grading were grade $2(n=16,47.05 \%)$ followed by grade $3(n=13$, $38.2 \%)$ and grade $1(\mathrm{n}=5,14.7 \%)$. Among 34 cases, 16 cases $(47.05 \%)$ had axillary lymph node metastasis and 8 cases $(23.5 \%)$ had a vascular invasion. Her2neu and HR phenotype cases were 11 each $(32.3 \%$ each) followed by 12 cases (35.2\%) of TNBC. 20 cases (58.8\%) had Ki67 $<20 \%$ and remaining cases $(\mathrm{n}=14,41.2 \%)$ had Ki67 $>20 \%$.

The mean value of TILs in CNB was $27.03 \%$ and $34.6 \%$ for the surgically resected specimen. The different parameters like age, subtype, grade, immunohistochemistry expression, vascular invasion were analyzed with respect to TILs between CNB and SRS. The mean value of TILs for the 
Table 1: Comparison of TILs score of CNB and resection specimens

\begin{tabular}{|c|c|c|c|c|c|}
\hline \multirow{2}{*}{ Parameters } & & \multicolumn{2}{|c|}{ Mean } & \multirow[b]{2}{*}{ Paired $\mathbf{t}$} & \multirow[b]{2}{*}{ P-value } \\
\hline & & CNB (\%) & SRS (\%) & & \\
\hline & Total & 27.03 & 34.68 & -2.5723 & 0.0148 \\
\hline \multirow{2}{*}{ Age Groups } & $\leq 50 \mathrm{yrs}$ & 24.41 & 37.56 & -2.9834 & 0.0088 \\
\hline & $\geq 51 \mathrm{yrs}$ & 29.65 & 31.79 & -0.5897 & 0.5636 \\
\hline ER & Negative & 36.06 & 43.97 & -1.9253 & 0.0722 \\
\hline \multirow{2}{*}{ PR } & Negative & 33.85 & 43.33 & -2.1540 & 0.0443 \\
\hline & Positive & 17.29 & 22.32 & -1.3864 & 0.1890 \\
\hline \multirow{2}{*}{ HER2 } & Negative & 28.23 & 31.27 & -1.1038 & 0.2822 \\
\hline & Positive & 24.83 & 40.92 & -2.5927 & 0.0250 \\
\hline Ki67 & $\leq 20$ & 24.21 & 32.45 & -2.5927 & 0.0250 \\
\hline \multirow{2}{*}{ Subtype } & IDC & 26.48 & 34.42 & -2.4784 & 0.0590 \\
\hline & Others & 32.67 & 37.33 & -0.6087 & 0.6047 \\
\hline \multirow{3}{*}{ Histological grade } & Grade 1 & 22.20 & 33.20 & -1.9318 & 0.1256 \\
\hline & Grade 2 & 23.50 & 31.31 & -1.8345 & 0.0865 \\
\hline & Grade 3 & 33.23 & 39.38 & -1.1060 & 0.2904 \\
\hline \multirow{3}{*}{ Phenotype classification } & HER2 & 26.27 & 43.23 & -2.5200 & 0.0304 \\
\hline & HR & 13.27 & 16.86 & -1.0741 & 0.3080 \\
\hline & TNBC & 40.33 & 43.17 & -0.6794 & 0.5109 \\
\hline \multirow{2}{*}{ Vascular Invasion } & Positive & 27.08 & 33.08 & -1.9281 & 0.0653 \\
\hline & Negative & 26.88 & 39.88 & -1.6879 & 0.1353 \\
\hline
\end{tabular}

Table 2: Distribution and agreement between of TILs scores between Biopsy and resection specimens (3-tiered)

\begin{tabular}{ccccc}
\hline CNBs & Low n (\%) & Intermediate n (\%) & High n (\%) & Total n (\%) \\
\hline Low & $3(8.82 \%)$ & $8(23.53 \%)$ & 0 & $11(32.35 \%)$ \\
Intermediate & $2(5.89 \%)$ & $9(26.47 \%)$ & $4(11.76 \%)$ & $15(44.12 \%)$ \\
High & 0 & $1(2.94 \%)$ & $7(20.59 \%)$ & $8(23.53 \%)$ \\
\hline Total & $\mathbf{5 ( 1 4 . 7 1 \% )}$ & $\mathbf{1 8 ( 5 2 . 9 4 \% )}$ & $\mathbf{1 1 ( 3 2 . 3 5 \% )}$ & $\mathbf{3 4}(\mathbf{1 0 0} \%)$ \\
\hline
\end{tabular}

Table 3: Distribution and agreement between of tumour-infiltrating scores between biopsy and resection specimens (2-tiered)

\begin{tabular}{cccc}
\hline CNBs & Low and Intermediate & High & Total \\
\hline Low and Intermediate & $22(64.7 \%)$ & $4(11.8 \%)$ & $26(76.5 \%)$ \\
\hline High & $1(2.9 \%)$ & $7(20.6 \%)$ & $8(23.5 \%)$ \\
\hline Total & $\mathbf{2 3}(\mathbf{6 7 . 6 \% )}$ & $\mathbf{1 1}(\mathbf{3 2 . 4 \% )}$ & $\mathbf{3 4}(\mathbf{1 0 0} \%)$ \\
\hline \multicolumn{4}{c}{}
\end{tabular}

young age group was $24.4 \%$ which was much lower than the scores in SRS (37.5\%). In elderly adults, the mean value of TILs for both age groups was quite close, which is $29.6 \%$ in CNB and $31.7 \%$ in SRS.

Infiltrating ductal carcinoma had a significant difference between TIL score of CNB (26.4\%) and SRS (34.4\%) in the present study. TIL score was higher in grade $3(33.23 \%)$ followed by grade $2(23.5 \%)$ tumours and grade 1 ( $22.2 \%$ ) tumours under CNB. But in SRS, grade 3 tumours $(39.38 \%)$ had more TIL followed by grade $1(33.2 \%)$ and grade $2(31.3 \%)$. Tumour with vascular invasion had low to intermediate TILs scores.
ER-positive and PR-positive tumour (HR phenotype tumour) had low to intermediate TILs score than ERnegative tumours in both CNB and SRS. HER 2 phenotypic tumours had a very low TIL score in CNB $(26.2 \%)$ than SRS (43.2\%). TNBC( 40.33\%) and HER2(26.27\%) phenotypic tumour showed a high TIL score against very low TIL values in HR phenotypic tumours (13.27\%). When low and high levels Ki67 percentage was compared, a TIL score of 24.21\% (low Ki67), 32.4\% (high Ki67) was encountered in CNB and 30.6\% (low Ki67), 37.5\% (high Ki67) TIL score in SRS.

On performing paired T-test upon the clinicopathological parameters between CNB TIL scores and SRS TIL scores, 
Table 4: Reliability of the TILs score in CNB with respect to the number of core

\begin{tabular}{|c|c|c|c|c|}
\hline Core Number & Number & Intraclass Correlation & F Test & P-Value \\
\hline 2 & 6 & 0.3440 & 1.5240 & 0.3270 \\
\hline $3-4$ & 7 & 0.9380 & 16.0680 & $0.0020 *$ \\
\hline 5 & 6 & 0.9690 & 32.7650 & $0.0010^{*}$ \\
\hline 6 & 7 & 0.9230 & 12.9680 & $0.0030 *$ \\
\hline $7 *$ & 8 & 0.8200 & 5.5410 & $0.0190 *$ \\
\hline
\end{tabular}
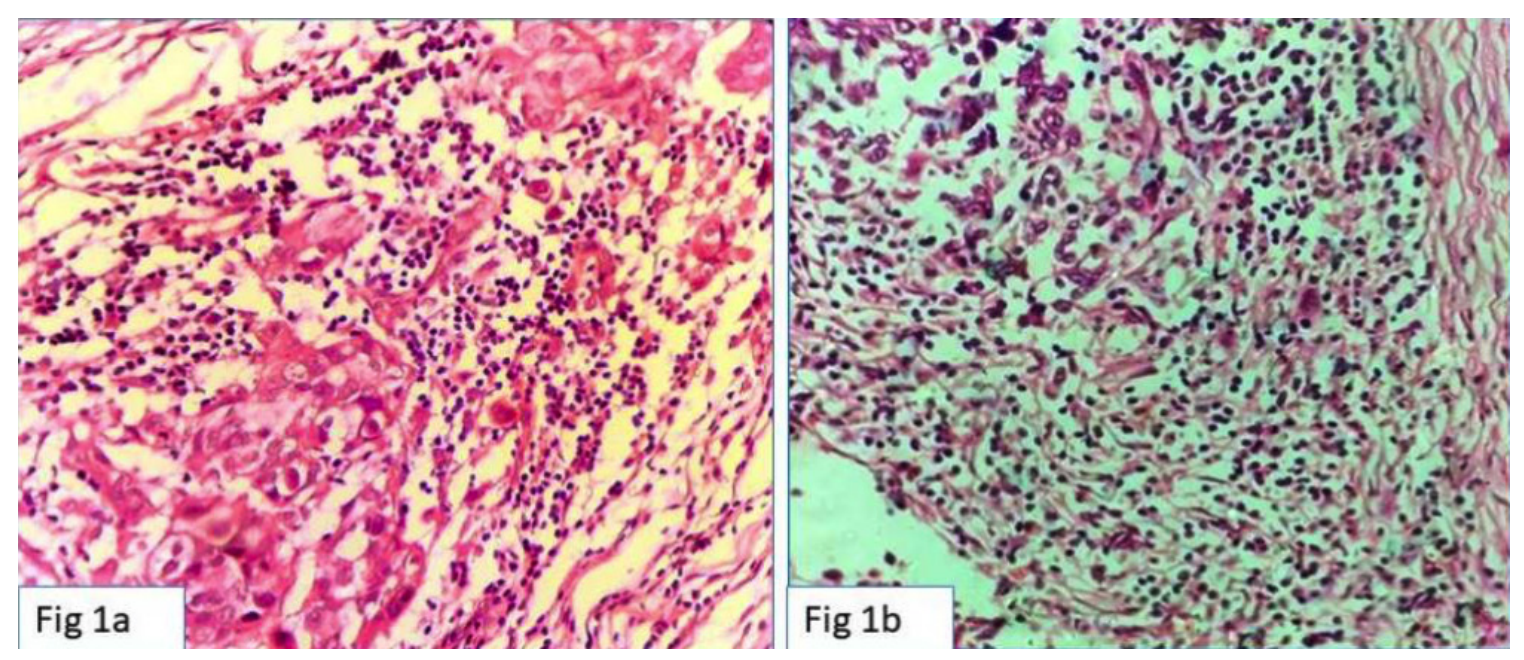

Figure 1: Case 1- TNBC phenotypic tumour showed TILs score on (a) CNB-70\% (b) SRS-62\% (HE-stain; X40)
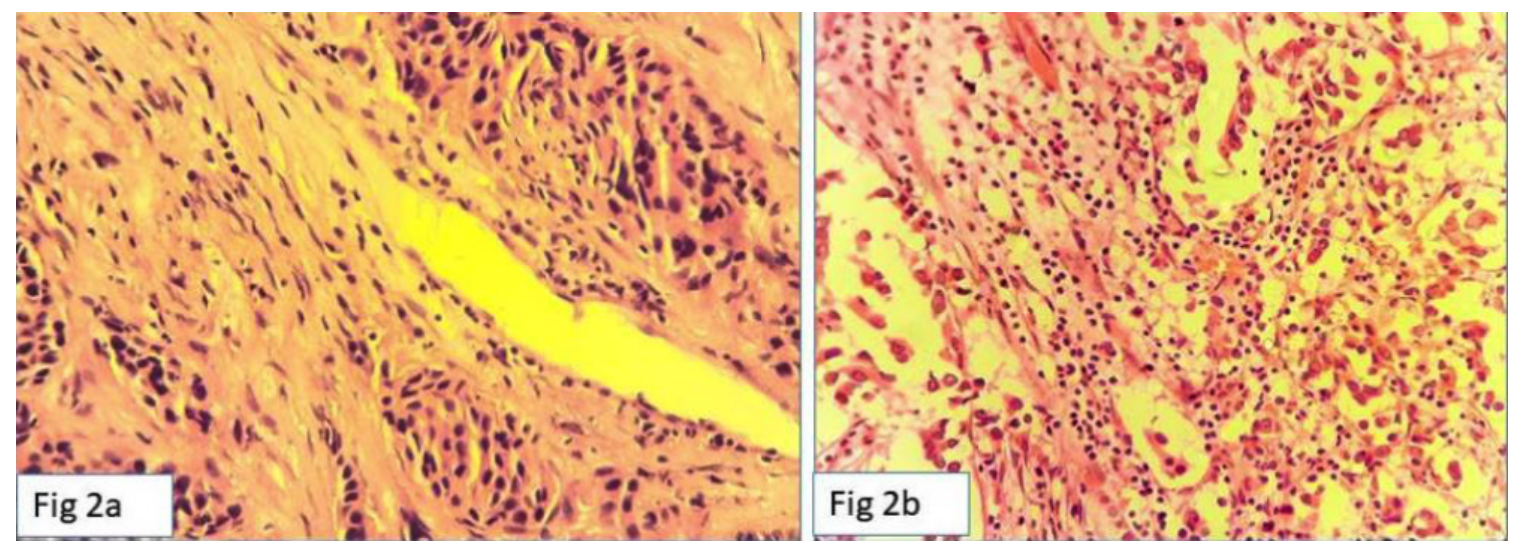

Figure. 2: Case 2- HR phenotypic tumour showed TILs score on (a) CNB- 9\% (b) SRS-15.5\% (HE-stain; X40)

we found that there was a statistically significant difference between TIL scores of CNB and SRS, with respect to a younger age, absence of PR and HER2 phenotypic tumours. (Table 1).

Intermediate (11-39\%) and high ( $>40 \%)$ TIL categories in CNB accounted for good agreement with SRS. The overall concordance rate was $27.2 \%$ for low, $60 \%$ for intermediate, and $87.5 \%$ for the high category. Out of 11 cases (32.3\%) which were put into the low TIL category on $\mathrm{CNB}, 8$ cases $(23.5 \%)$ turned out to be in intermediate, none in high, and the remaining 3 cases $(8.82 \%)$ in the low category after SRS. In the intermediate category, out of 15 cases (44.1\%), most were in intermediate $(\mathrm{n}=9,26.47 \%), 4$ cases $(11.7 \%)$ in high, and 2 cases $(5.89 \%)$ in the low category. In the high category, out of 8 cases $(23.5 \%)$, one $(2.94 \%)$ was in intermediate, and the remaining 7 cases $(20.5 \%)$ coincided with a high TIL score with regards to SRS. There was a 77.94 $\%$ kappa agreement between three categories $(\mathrm{k}=0.43)$.

When we merged low and intermediate and made a 2 tier system, the agreement improved to $82 \%$ between CNB and SRS. The concordance rate of the combined low and intermediate category improved to $81.4 \%$. (Table 3 )

The ICC (r) of these variables were 0.7 in all cases, 0.4 for HER2 $(n=11), 0.3$ for HR $(n=11)$ and $0.8(n=12)$ for TNBC. A r-value of 0.0-0.2 correlates with slight, 0.1-0.4 with fair , 0.41-0.6 with moderate, $0.6-0.8$ with substantial and 0.81 with near perfect. The ICC values for hormone expression 


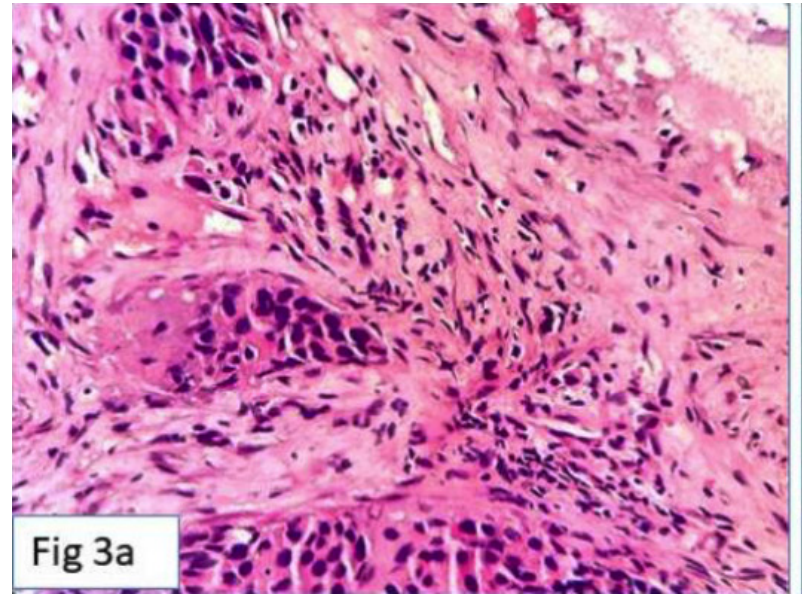

Figure3: Case 3- HER2 phenotypic tumour showed TILs score on (a) CNB-15\%

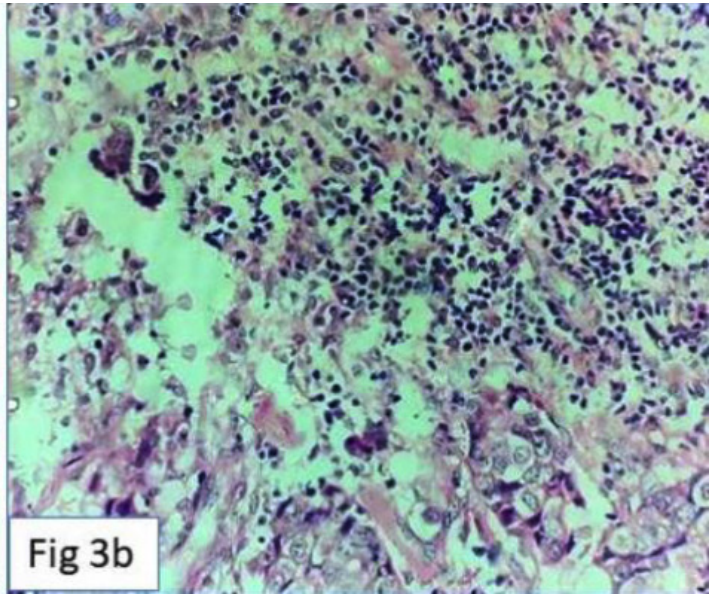

(b) SRS- 74\% (HE-stain; X40). were categorised from fair to near perfect levels $(0.3-0.81)$ in present study.

After univariate analysis, the parameters which had confounding factors that affected the TIL score were age, PR status, Her2 phenotypic tumour, and the number of cores. Our study showed that more number of cores $(\geq 3)$ in CNB was significantly associated with a smaller difference in the TIL score. (Table 4)

On comparing the TIL score with respect to phenotype classification of breast carcinoma by ANOVA, the mean TIL score in HER2, HR, TNBC was lower in CNB than the SRS and the difference was statistically significant. The difference in TIL score between HR and HER2 is not significant in CNB but is significant in SRS. Similarly, the difference in TILs score between HR and TNBC is significant in both CNB and SRS. The difference of TIL score in HER2 versus TNBC is not significant in both CNB and SRS since both types of tumours have a high TIL score. The microphotographs of the TIL distribution in various phenotypic tumours are explained (fig. 1-3).

\section{DISCUSSION}

The present study examined the clinicopathological profiles of TILs in patients with primary breast carcinoma. We found that there was a considerable disparity between TIL score of CNB and SRS with younger age ( $<50$ years), PR status, and HER2 phenotype tumours. CNB is expected to be composed of tissue from the periphery of the tumour and less from the centre. ${ }^{20}$ Few studies hold an opinion that tissue with high microvessel density sets off more immune activation which brings about more hypoxia and necrotic change in the malignant tumours. ${ }^{26}$ These are the rationale behind the difference in CNB and SRS TIL score. The present study and the study done by Cha et al support this hypothesis as we have got a lower mean TIL score for CNBs than the mean TIL score in SRS.
On analyzing the scores at a young age, the antecedent cause was firstly CNB procedure elicits an immune response in breast cancer which justifies the reason for the difference in TIL score between the two. ${ }^{26-28}$ The TIL change after CNB between young and elderly is significant because the immune activation in the elderly is subtle when compared to younger patients because of a decrease in immune function, degeneration of thymus gland, less recruitment of T cell. ${ }^{29}$ Thus, TIL is more consistent and uniformly distributed in the elderly than young patients. Cha et al also showed that there was less change in elderly patients between CNB and SRS. ${ }^{30}$

TIL score when compared between different grades of the tumour showed a difference between CNB and SRS. The reason may be while performing histological grading of the tumour on CNBs there is a propensity to under categorize the tumour by virtue of underestimating the mitotic counts during biopsy and overestimation of overall nuclear grade on SRS. ${ }^{20}$

Our study showed TIL score was low in the majority of cases and intermediate category for ER/PR positive tumours. Loi et al showed that there is lower activation of TILs in ERpositive tumours than triple-negative tumours. ${ }^{31}$ Cha et al showed that ER/PR positive tumour had an intermediate mean score for TIL. ${ }^{30}$ PR positivity is heterogeneous in the tumour when compared to ER positivity which is more homogenously stained. ${ }^{32}$ Therefore PR positivity acts as a confounding factor for TIL scoring $(\mathrm{p}<0.05)$.

Our study unveils that there is no correlation in the TIL score between CNB and SRS in tumours with HER2 phenotype. HER2 subtypes tumours show a higher level of intratumoral heterogeneity in TIL. But a study done by Cha et al showed there is near perfect reliability between biopsy and SRS in HER2 phenotypic tumours against fair reliability in our study. ${ }^{30}$ Our study showed that the variables responsible for discordance were younger age, HER2 status, and PR status. The present study showed that the correlation between CNB 
and SRS improved for $\geq 3$ cores. Cha et al proved that $\geq 5$ cores are sufficient to get good agreement between CNB and SRS.

The limitations of the study are firstly the sample size is very small. In the phenotypic sub-classification of breast cancers where the essence of the study lies in the era of chemotherapy, there is an uneven distribution of cases. This can lead to uncertain results.

More studies are required to evaluate spatial and temporal heterogeneity in breast cancer by tissue microarrays and immunohistochemistry (CD8, FOXP3). More studies with complete follow-up along with the determination of overall survival and disease-free survival with neoadjuvant chemotherapy, immunomodulation therapies with regard to TIL is required. Recommendations by an International TILs Working Group 2014 while dealing with tumour with high TIL heterogeneity should be reconsidered.

\section{CONCLUSIONS}

TIL score in CNB is of reliable value in breast cancer. Discrepancies may occur in tumours with HER2 phenotype, PR status and younger age. More than three cores may accurately predict the TIL score.

\section{Conflict of interest: None}

\section{REFERENCES}

1. Malyguine AM, Strobl SL, Shurin MR. Immunological monitoring of the tumor immune environment for clinical trials. CancerImmunol Immunother 2012;61:239-47. Crossref

2. Lal P, Tan LK, Chen B. Correlation of HER-2 status with estrogen and progesterone receptors and histologic features in 3,655 invasive breast carcinomas. Am J ClinPathol 2005;123:541-6. Crossref

3. Vakkila J, Lotze MT. Inflammation and necrosis promote tumour growth. Nat Rev Immunol 2004;4:641-8. $\underline{\text { Crossref }}$

4. DeNardo DG, Coussens LM. Inflammation and breast cancer. Balancing immune response: crosstalk between adaptive and innate immune cells during breast cancer progression. Breast Cancer Res 2007;9:212. Crossref

5. Freedman RS, Vadhan-Raj S, Butts C, et al. Pilot study of FLT3 ligand comparing intraperitoneal with subcutaneous routes on hematologic and immunologic responses in patients with peritoneal carcinomatosis and mesotheliomas. Clin Cancer Res 2005;9:522837. Crossref

6. Melichar B, Freedman RS. Immunology of the peritoneal cavity: Relevance for host-tumor relation. Int J Gynecol Cancer 2002;12:317. Crossref

7. Melichar B, Nash MA, Lenzi R, et al. Expression of costimulatory molecules $\mathrm{CD} 80$ and $\mathrm{CD} 86$ and their receptors CD28, CTLA-4 on malignant ascites CD3+ tumor-infiltrating lymphocytes (TIL) from patients with ovarian and other types of peritoneal carcinomatosis. ClinExpImmunol 2000;9:19-27. Crossref
8. Melichar B, Savary CA, Patenia R, et al. Phenotype and antitumor activity of ascetic fluid monocytes in patients with ovarian carcinoma. Int J Gynecol Cancer 2003;13:435-43. Crossref

9. Kato T, Kameoka S, Kimura $\mathrm{T}$, et al. The combination of angiogenesis and blood vessel invasion as a prognostic indicator in primary breast cancer. Br J Cancer 2003;88:1900-8. $\underline{\text { Crossref }}$

10. Lee AK, DeLellis RA, Silverman ML, et al. Prognostic significance of peritumoral lymphatic and blood vessel invasion in nodenegative carcinoma of the breast. J ClinOncol 1990;8: 1457-65. $\underline{\text { Crossref }}$

11. Mohammed ZMA, Going JJ, Edwards J, et al. The relationship between components of tumour inflammatory cell infiltrate and clinicopathological factors and survival in patients with primary operable invasive ductal breast cancer. $\mathrm{Br} \mathrm{J}$ Cancer [Internet] 2012;107:864-73. Crossref

12. Loi S, Michiels S, Salgado $\mathrm{R}$, et al. Tumor infiltrating lymphocytes are prognostic in triple negative breast cancer and predictive for trastuzumab benefit in early breast cancer: results from the FinHER trial. Ann Oncol 2014;25:1544-50. Crossref

13. Adams S, Gray RJ, Demaria S, et al. Prognostic value of tumorinfiltrating lymphocytes in triple-negative breast cancers from two phase III randomized adjuvant breast cancer trials. J Clin Oncol 2014;32:2959-66. Crossref

14. Loi S, Sirtaine N, Piette F, et al. Prognostic and predictive value of tumor-infiltrating lymphocytes in a phase III randomized adjuvant breast cancer trial in node-positive breast cancer comparing the addition of docetaxel to doxorubicin with doxorubicinbased chemotherapy: BIG 02-98. J ClinOncol 2013;31:860-7. Crossref

15. Pruneri G, Vingiani A, Bagnardi V, et al. Clinical validity of tumor-infiltrating lymphocytes analysis in patients with triple-negative breast cancer. Ann Oncol 2016;27:249-56. Crossref

16. Denkert C, Loibl S, Noske A, et al. Tumor-associated lymphocytes as an independent predictor of response to neoadjuvant chemotherapy in breast cancer. J ClinOncol 2010;28:105-13. Crossref

17. Denkert C, von Minckwitz G, Brase JC, et al. Tumor-infiltrating lymphocytes and response to neoadjuvant chemotherapy with or without carboplatin in human epidermal growth factor receptor 2-positive and triple-negative primary breast cancers. J ClinOncol 2015;33:983-91. $\underline{\text { Crossref }}$

18. Salgado R, Denkert C, Campbell C, et al. Tumor-Infiltrating Lymphocytes and Associations With Pathological Complete Response and Event-Free Survival in HER2-Positive Early-Stage Breast Cancer Treated With Lapatinib and Trastuzumab: A Secondary Analysis of the NeoALTTO Trial. JAMA Oncol 2015;1:448-54. $\underline{\text { Crossref }}$

19. Heppner BI, Untch M, Denkert C, et al. Tumor-Infiltrating Lymphocytes: A Predictive and Prognostic Biomarker in Neoadjuvant-Treated HER2-Positive Breast Cancer. Clin Cancer Res 2016;22:5747-54. $\underline{\text { Crossref }}$

20. Huang J, Chen X, Fei X, et al. Changes of Tumor Infiltrating Lymphocytes after Core Needle Biopsy and the Prognostic Implications in Early Stage Breast Cancer: A Retrospective Study. Cancer Res Treat 2014;51:1336-46. $\underline{\text { Crossref }}$

21. Lee KH, Kim EY, Yun JS, et al. The prognostic and predictive value of tumor-infiltrating lymphocytes and hematologic parameters in patients with breast cancer. BMC Cancer 2018;18:938. Crossref 
22. Arnedos M, Nerurkar A, Osin P, et al. Discordance between core needle biopsy $(\mathrm{CNB})$ and excisional biopsy (EB) for estrogen receptor (ER), progesterone receptor (PgR) and HER2 status in early breast cancer (EBC). Ann Oncol 2009;20:1948-52. Crossref

23. Chen $\mathrm{X}$, Zhu S, Fei $\mathrm{X}$, et al. Surgery time interval and molecular subtype may influence Ki67 change after core needle biopsy in breast cancer patients. BMC Cancer 2015;15:822. Crossref

24. Salgado R, Denkert C, Demaria S. The evaluation of tumorinfiltrating lymphocytes (TILs) in breast cancer: recommendations by an International TILs Working Group 2014. Ann Oncol 2015;26:259-71. Crossref

25. Goldhirsch A, Winer EP, Coates AS, et al. Personalizing the treatment of women with early breast cancer: highlights of the St Gallen International Expert Consensus on the Primary Therapy of Early Breast Cancer Ann Oncol. 2013;24:2206-23. $\underline{\text { Crossref }}$

26. Vaupel P, Mayer A. Tumor Oxygenation Status: Facts and Fallacies. Adv Exp Med Biol 2017;977:91-9. Crossref

27. Jeselsohn RM, Werner L, Regan MM, et al. Digital quantification of gene expression in sequential breast cancer biopsies reveals activation of an immune response. PLoS One 2013;8:e64225. Crossref
28. Mathenge EG, Dean CA, Clements D, et al. Core needle biopsy of breast cancer tumors increases distant metastases in a mouse model. Neoplasia. 2014;16:950-60. Crossref

29. Globerson A, Effros RB. Ageing of lymphocytes and lymphocytes in the aged. Immunol Today. 2000;21:515-21. Crossref

30. Cha YJ, Ahn SGGG, Bae SJ, et al.Comparison of tumor-infiltrating lymphocytes of breast cancer in core needle biopsies and resected specimens: a retrospective analysis Breast Canc Res Treat 2018;171:295-302. Crossref

31. Loi S. Tumorinfiltrating lymphocytes, breast cancer subtypes and therapeutic efficacy. Oncoimmunology 2013;2:e24720. Crossref

32. Zidan A, Christie Brown JS, Peston D, et al. Oestrogen and progesterone receptor assessment in core biopsy specimens of breast carcinoma. J ClinPathol 1997;50:27-9. Crossref 